\title{
Circuit
}

Musiques contemporaines

\section{L’archéologie imaginaire de François-Bernard Mâche : fouille de ses écrits}

\section{Sharon Kanach}

Volume 29, numéro 1, 2019

URI : https://id.erudit.org/iderudit/1059433ar

DOI : https://doi.org/10.7202/1059433ar

Aller au sommaire du numéro

Éditeur(s)

Circuit, musiques contemporaines

ISSN

1183-1693 (imprimé)

1488-9692 (numérique)

Découvrir la revue

Citer ce document

Kanach, S. (2019). L'archéologie imaginaire de François-Bernard Mâche :

fouille de ses écrits. Circuit, 29(1), 83-86. https://doi.org/10.7202/1059433ar d'utilisation que vous pouvez consulter en ligne.

https://apropos.erudit.org/fr/usagers/politique-dutilisation/ 


\section{L'archéologie imaginaire de François- Bernard Mâche : fouille de ses écrits}

Sharon Kanach

Quand le mythe meurt, la musique devient mythique de la même façon que les œuvres d'art, quand la religion meurt, cessent d'être simplement belles pour devenir sacrées.

- Claude Lévi-Strauss ${ }^{1}$

La triple vie (et carrière) de François-Bernard Mâche (1935) en tant que compositeur, universitaire et philosophe de la musique est rare de par son ampleur, sa réussite, et aussi, hélas, un certain manque de reconnaissance, tant à l'international que dans son pays natal. C'est pourquoi souffler ses 80 bougies a du bon, notamment pour ceux qui doivent si injustement attendre un tel événement pour que la lumière se focalise finalement sur eux. Mais grâce à cela, nous avons assisté récemment à des publications à foison et des hommages en tous genres. Enfin! C'est aussi le parfait prétexte pour replonger dans les travaux et réalisations antérieurs de Mâche, car il faut faire beaucoup de chemin pour aboutir à une œuvre si riche, innovante, magnifique.

Issu d'une famille de musiciens, le jeune FrançoisBernard choisit, malgré son talent musical précoce, d'entreprendre des études universitaires des plus ardues: dans la même "prépa» au lycée Louis-leGrand que Jean-Claude Risset, Daniel Charles et Alain Badiou, parmi d'autres, il entre ensuite à la prestigieuse École normale supérieure. Il y obtient son diplôme d'archéologie grecque en 1957 et sort agrégé de lettres classiques (latin, grec, français) l'année suivante. En 1958, il passe également le concours d'entrée au Conservatoire de Paris afin d'y suivre les cours d'Olivier Messiaen, notamment, tout en cofondant le Groupe de recherches musicales (GRM), dont il devient, ponctuellement, le directeur pour l'année 1962. Grâce à l'enseignement de Messiaen, Mâche reçoit son premier Prix de philosophie de la musique. Et philosophe de la musique, François-Bernard Mâche l'est resté toute sa vie, comme on le constate à la lecture de ses très nombreux écrits, que nous revisitons ici pour certains, et découvrons pour d'autres. Bien plus tard, en 1980, il soutient son doctorat d'État dont le sujet est l'idée de modèle en musique. Son directeur de thèse est Iannis Xenakis, son ami de toujours, un autre grand philosophe de la musique et, peut-être, l'exemple que le benjamin souhaitait émuler, pour qui également la musique est connaissance.

En tant qu'universitaire, son parcours est un parangon de rigueur et de patience. Parmi ses premiers postes, Mâche enseigne le latin et le grec à son alma mater, Louis-le-Grand, réputé pour être le meilleur lycée de France, jusqu'en 1982 (et sans doute en profite-t-il pour, parallèlement, poursuivre sa recherche sur les langues mortes ou en voie de disparition qui lui sont si chères, musicalement). C'est à l'âge de 48 ans qu'il entame sa carrière de professeur de musicologie 
à l'Université de Strasbourg où, d'ailleurs, il crée le Centre PRIMus où est proposé le premier cursus pour des ingénieurs du son en France. En 1993, il est élu directeur d'études à l'École des hautes études en sciences sociales avec un programme de recherche consacré aux « universaux musicaux». Tout est dit.

Malgré la crainte que sa formation intellectuelle soit « un handicap pour une carrière artistique véritable²», et en parallèle à son investissement considérable sur le plan universitaire, Mâche travaille avec acharnement au développement de son propre langage musical, personnel et distinct, celui qu'il appelle «la troisième voie» : celle de la nature comme modèle et comme matériau. Pour ce faire, il travaille à la composition, certes, mais également à une constante réflexion scientifique qu'il élabore dans ses écrits : ses premières œuvres musicales reconnues datent de 1957 et ses premiers écrits, de 1959 ( Connaissance des structures sonores» et «Synthèse expérimentale»)

Deux parutions récentes et d'autres rééditions nous offrent l'occasion de plonger en profondeur dans «l'archéologie imaginaire » de François-Bernard Mâche. À ce jour, avec quelque 113 opus à son catalogue (allant de solos aux grandes œuvres pour orchestre, avec un réel penchant pour l'électroacoustique), son œuvre peut non seulement s'écouter, mais aussi se lire en regards croisés au travers de ses écrits, encore plus nombreux. Bien entendu, rien ne vaut autant que la voix du maître, mais les très récents actes de colloque François-Bernard Mâche: le compositeur et le savant face à l'univers sonore (sous la direction de Márta Grabócz et de Geneviève Mathon, Paris, Hermann, 2018, 418 pages) peuvent servir de guide pour éclairer les pistes vers des textes précis du compositeur.

De plus, ce livre est accompagné d'un DvD contenant des extraits du colloque et des tables rondes tenus en octobre 2015 (presque sous le même titre que le livre, seul le mot «compositeur» est remplacé par

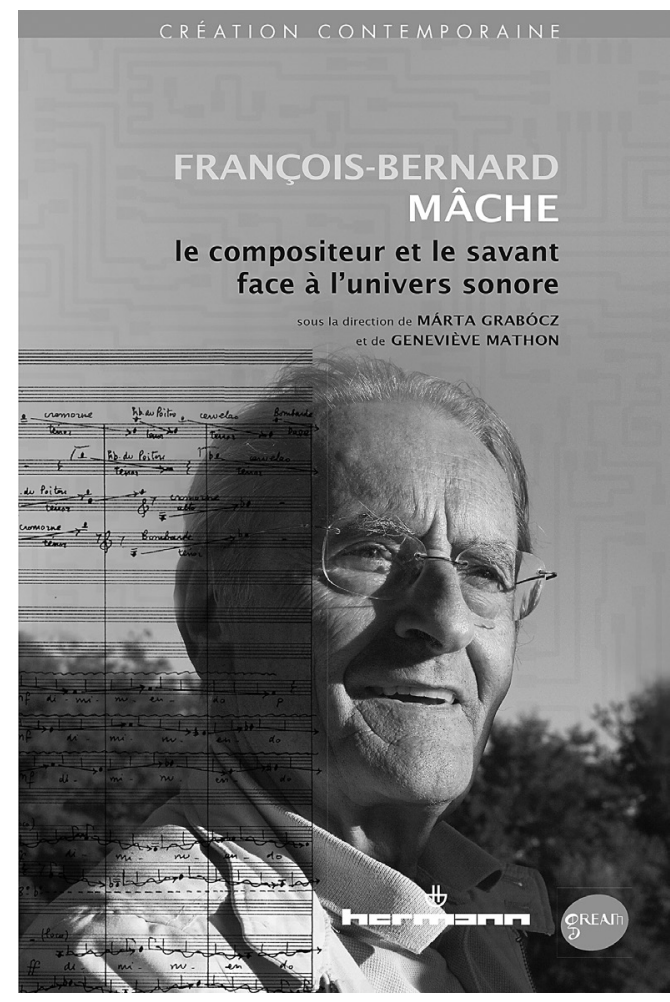

concert donné à Strasbourg par l'ensemble Accroche Note. Après l'excellente et synthétique introduction de la grande spécialiste de Mâche, Márta Grabócz, ce livre est divisé en cinq parties: «Esthétique de la troisième voie, l'universel et le particulier » (sept articles); «Zoomusicologie et chants d'oiseaux » (cinq articles, dont deux en anglais); "Archétypes, Modèles et dialogue des civilisations » (trois articles); «Écologie sonore et écho-mythologie » (deux articles); «Musiques mixtes, musique spectrale» (cinq articles), pour un total de vingt-deux contributions (dont deux en anglais). Le sommaire du DVD (réalisé lors de la résidence de Mâche à Strasbourg en 2015) comprend le concert donné le $1^{\text {er }}$ décembre 2015 par l'ensemble Accroche Note et les élèves du conservatoire de Strasbourg (six œuvres allant de 1982 à 2007), une 


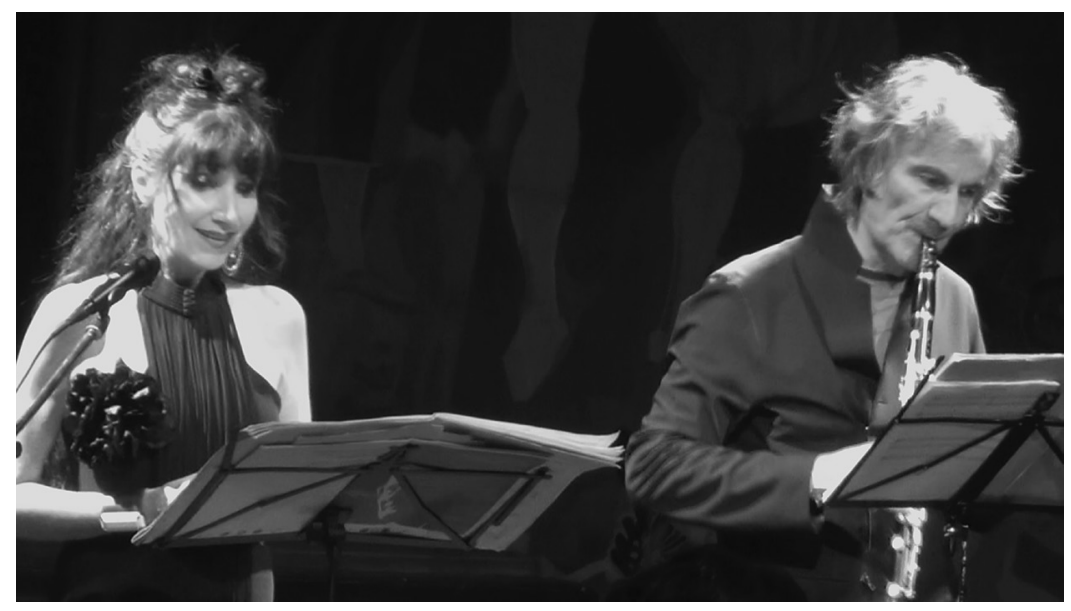

Aliunde en concert, ensemble Accroche Note, Françoise Kubler et Armand Angster, 10 octobre 2015, Paris. (C) L. Pottier. (Laurent Pottier in Grabócz et Mathon [dir.], 2018, p. 336.)

rencontre entre le compositeur et certains contributeurs au livre plus Armand Angster (clarinettes) et Françoise Kubler (voix), onze extraits d'une table ronde du 10 octobre 2015 avec des compositeurs autour de Mâche, incluant, entre autres, deux belles interventions de feu Jean-Claude Risset.

Par ailleurs, les vidéos complètes des classes de maître sur deux œuvres données au concert par les élèves du Conservatoire de Strasbourg sont désormais disponibles sur Internet: il s'agit de Figures (1989), pour clarinette basse et vibraphone, et de Trois chants sacrés (1982-1990), pour voix soliste ${ }^{4}$. Ces cinq sections démarquent bien les préoccupations à la fois musicales et théoriques de notre compositeur-savant-poète et se nourrissent de travaux scientifiques au plan d'arête sans trop rentrer dans une hagiographie démesurée. De plus, un joli dialogue s'instaure entre le DVD et les textes, donnant un visage - littéralement - humain à cette collection.

Le deuxième ouvrage sorti tout dernièrement est de François-Bernard Mâche lui-même: Le sonore et l'universel: écrits au tournant du XXI e siècle (Paris, Éditions des archives contemporaines, 2018, 354 pages).
Ce livre est divisé en six grandes parties: «La création artistique » (onze entrées) ; «Langage et musique » (six entrées); «La nature et le musicien » (neuf entrées); «Musicologie» (sept entrées, dont deux en anglais); «Regards sur d'autres musiciens»(dix entrées); «Techniques» (sept entrées), pour un impressionnant total de cinquante et une entrées, précédé d'une courte introduction de la même Márta Grabócz et d'un bref avant-propos de l'auteur. Ces écrits, pour la plupart assez brefs, datent de 1994 jusqu'à 2017 et traitent parfois de sujets inédits pour Mâche, mais ils servent surtout à préciser, à peaufiner sa pensée déjà exprimée dans des publications antérieures, comme MusiqueMythe-Nature de 1983, magistralement rééditée pour la deuxième fois en 2015 par le courageux éditeur parisien Ædam Musicæ5. Cette nouvelle édition, tout en regroupant les cinq textes fondamentaux de la philosophie et de la démarche mâchiennes («La musique dans le mythe »; «Universalité des modèles sonores»; «Langage et Musique»; «Zoomusicologie»; «Le Modèle en musique») est considérablement plus annotée que les deux précédentes éditions (et les soixantehuit exemples cités sont présents sur un généreux CD 


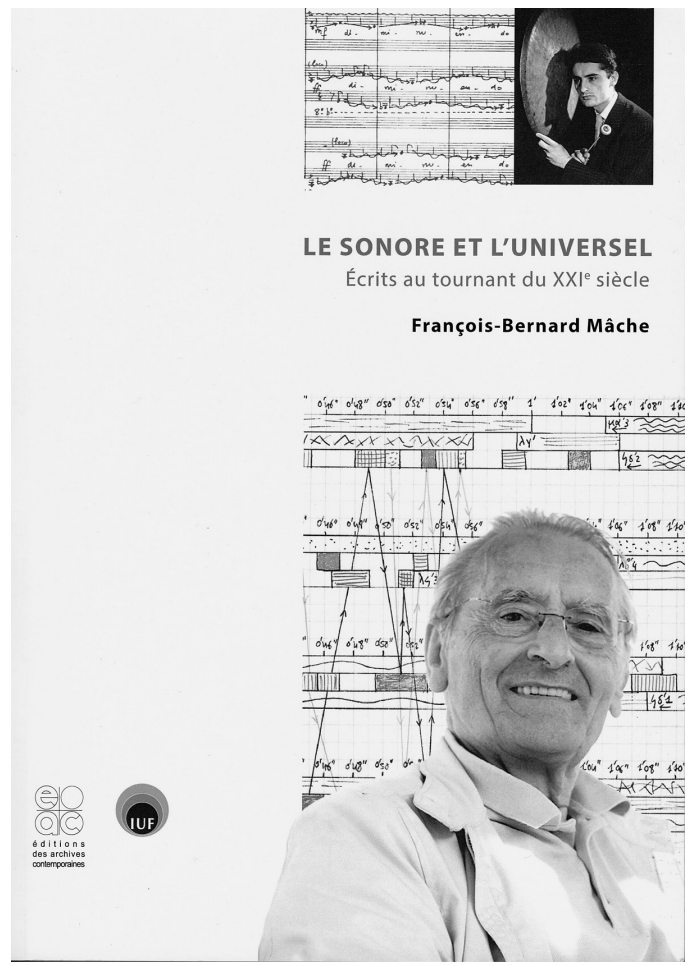

d'extraits sonores) et les textes sont parfois remaniés, principalement dans leur forme, mais aussi dans leur contenu. Le livre où Mâche se révèle, à notre avis, le plus poète, est Musique au singulier (Paris, Odile Jacob, 2001, 310 pages). Reprenant des thèmes omniprésents dans l'univers mâchien comme les archétypes, l'universel en musique, le mythe, la nature et les «polyphonies animales ", Mâche, ici, se permet d'aborder l'émotion, tant à l'écoute qu'au maniement des sons.

Autre ouvrage de François-Bernard Mâche, Un demisiècle de musique... et toujours contemporaine (Paris, L'Harmattan, 2000, 434 pages) est aussi un complément indispensable à Musique-Mythe-Nature et à Musique au singulier, car il recoupe un très large ensemble d'écrits (soixante-dix entrées!) datant de 1959 à 1999, organisés non pas par thème, mais simplement chronologiquement, qui ponctuent et renforcent ses préoccupations
Pour aborder l'homme en chair et os, et avec une sincérité parfois déroutante ("mais je n’ai jamais été très sociable», p. 8), nous conseillons l'excellent livre d'entretiens avec le journaliste Bruno Serrou: De la musique, des langues et des oiseaux (Paris, Michel de Maule, 2007, 316 pages, accompagné d'un DVD avec l'intégral de l'entretien entre les deux hommes presque onze heures enregistrées en décembre 2004!).

Et pour accéder à la belle musique de FrançoisBernard Mâche, d'une manière originale et provocatrice, on consultera son indispensable Cent opus et leurs échos (Paris, L’Harmattan, 2012, 324 pages, préfacé par l'incontournable Márta Grabócz et avec une courte introduction par le compositeur). Comme son titre l'indique, cet ouvrage fait état des cent premières œuvres de Mâche (sur 113 actuellement), allant de son premier Duo (1957) pour violon et piano jusqu'à son Le promeneur solitaire (2010), un «autoportrait» électroacoustique. Titre par titre, le compositeur donne les «Notices d'origine» sur ses œuvres, très souvent complétées par des «Commentaires» réactualisés, et, lorsqu'elles sont disponibles, un choix de critiques parues dans la presse, certaines élogieuses, d'autres plus contestataires. Un tel recueil chronologique propose ainsi une véritable lecture sociologique de la musique française de la deuxième moitié du $\mathrm{Xx}^{\mathrm{e}}$ siècle et du début du XXI ${ }^{\mathrm{e}}$, à travers le prisme d'un de ses compositeurs les plus féconds.

\footnotetext{
1. Claude Lévi-Strauss (1971), Mythologiques 4: L'homme nu, Paris, Plon, p. 584.

2. François-Bernard Mâche (2007), De la musique, des langues et des oiseaux: entretien avec Bruno Serrou, Paris, Michel de Maule, p. 50.

3. François-Bernard Mâche (2000), Un demi-siècle de musique... et toujours contemporaine, Paris, L'Harmattan.

4. L'ouvrage indique (p. 406) les liens: http://utv.unistra.fr/video. php?id_video $=986$ et http://utv.unistra.fr/video.php?id_video $=987$ (consultés le 16 décembre 2018).

5. François-Bernard Mâche (2015[1983]), Musique-Mythe-Nature, Paris, Ædam Musicæ.
} 